\title{
Electrospun polystyrene fibers for HIV entrapment
}

Chaobo Huang ${ }^{1, a}$, Stefaan J. Soenen ${ }^{2, a}$, Ellen van Gulck ${ }^{3}$, Joanna Rejman ${ }^{2}$, Guido Vanham ${ }^{3,4}$, Bart Lacus ${ }^{2}$, Bart Geers ${ }^{2}$, Kevin Braeckmans ${ }^{2}$, Victor Shahin ${ }^{5}$, Pieter Spanoghe ${ }^{6}$, Jo Demeester ${ }^{2}$, Stefaan C. De Smedt ${ }^{2} *$

1. College of Chemical Engineering, Nanjing Forestry University (NFU), Nanjing 210037, P. R. China

2. Lab of General Biochemistry and Physical Pharmacy, Faculty of Pharmaceutical Sciences, Ghent University, Harelbekestraat 72, B9000 Ghent Belgium

3. Virology Unit, Department of Microbiology, Institute of Tropical Medicine, Belgium

4. Department of Biomedical Sciences, Faculty of Pharmacology, Biomedical and Veterinary Sciences, University of Antwerp, Faculty of Medicine and Pharmacy, Belgium

5. Institute of Physiology II, University of Münster, Münster, Germany.

6. Department of Crop Protection Chemistry, Ghent University, Ghent, Belgium

a These authors contributed equally to this work

* Corresponding author: Tel.: +3292648076

Fax: +32 92648189

Email: Stefaan.DeSmedt@UGent.be

\begin{abstract}
:
The high versatility and ease of electrospinning of polymer solutions have recently resulted in electrospun fibers which are of interest for a wide variety of chemical and biomedical applications. This is partially due to the high surface area of the fibers which is attractive for the detection and capture of (bio)chemicals. In the present work, polystyrene (PS) fibers were electrospun and coated with cationic poly(allylamine hydrochloride) (PAH) or anionic dextran sulfate (DSS). The fibers were physicochemically characterized. Upon incubation in a dispersion of inactivate HIV-1, avid binding of HIV to all types of fibers occurred. By atomic force microscopy and spatial selective photobleaching, the binding of the inactivate HIV-1 particles to the fibers could be confirmed. Interestingly, all fibers but especially the DSS- and PAH-coated ones resulted in a significant reduction of infection of $\mathrm{CD}^{+} \mathrm{TZMb} 1$ cells by replication-competent HIV-1. On top, DSS-coated PS fibers were not toxic for vaginal epithelial cells which may make these fibers of potential interest to inhibit HIV infection in the context of topical prevention.
\end{abstract}




\section{Introduction}

Electrospinning is gaining widespread attention as it is generally considered as one of the most facile methods to produce a wide range of nano- and microsized polymeric fibers. ${ }^{1}$ The basic process of electrospinning consists of a polymer solution or melt which is pumped through a needle which is connected to a high voltage source (Figure 1A). Due to the electrostatic forces generated, whipped polymer jets will be created at the needle tip. These jets can then be caught on a grounded collector, the remaining solvent evaporates and leaves naked electrospun fibers in a dry state. ${ }^{2}$ By modification of several processing parameters (voltage applied, conductivity and viscosity of the polymer solution, flow rate and distance between the needle tip and the collector), ${ }^{2}$ the porosity and size of the fibers (diameter commonly ranging from $100 \mathrm{~nm}$ to several micrometers with lengths of several millimeters) can be easily adapted to better suit the envisaged applications. As there are no stringent requirements regarding the type of polymer used, the process of electrospinning can be used to generate fibers consisting of a wide array of polymers, either natural or synthetic ones. ${ }^{3,4}$ Furthermore, more advanced setups are also available, such as coaxial spinning, to produce core/shell fibers, and the use of a rotating drum collector to generate highly aligned fibers ${ }^{5,6}$.

The high number of variables made possible by the electrospinning process has resulted in a wide variety of applications, primarily in the biomedical field: (a) first electrospun fibers are often investigated as scaffolds for tissue engineering applications ${ }^{7-9}$ (b) the wide array of polymers used, including stimulus-sensitive ones, conjoined with the ease of encapsulation of a wide variety of compounds, has resulted in a high interest in electrospun fibers for advanced drug delivery applications; ${ }^{10-12}$ (c) furthermore, the ability to control fiber alignment has resulted in the use of electrospun fibers as tools to guide stem cell differentiation; ${ }^{13-15}$ (d) the high porosity and specific surface area of electrospun fibers result in a high availability for compounds in the surrounding medium to binding sites on the fibers. ${ }^{4}$ This property has been exploited to develop chemical sensors, with high sensitivity and very fast reaction times ${ }^{16,17}$ and suggests the possible use of fibers for binding and removal of toxins or pathogens; (e) other applications include the encapsulation of living cells and single cell organisms, ${ }^{18}$ the labeling of pharmaceutical tablets against counterfeiting with encoded electrospun fibers ${ }^{19}$ 
and the design of scaffolds for wound dressing. ${ }^{20}$

We became interested to test the ability of electrospun fibers to capture HIV viral particles as it could become an attractive strategy to prevent HIV infection. In a first series of experiments we observed, by co-incidence, avid binding of HIV viral particles to the walls of polystyrene (PS) and polypropylene (PP) Eppendorf tubes; This presumably non-specific binding of inactivated HIV, dispersed in a simulated vaginal fluid (SVF) with a $\mathrm{pH}$ of 4.2 , to PS and PP eppendorf tubes was quantified using an ELISA assay against the viral protein p24. After $2 \mathrm{~h}$ of incubation, about $25 \%$ of the viral particles became bound to the PP tubes wile binding to the PS tubes was even about $35 \%$. This (unexpected) observation inspired us to test the ability of PS and PP electrospun fibers to bind inactivated HIV viral particles. Being aware of the fact that polyelectrolytes (like polystyrene sulfonate) are under investigation as microbicides (to be applied intravaginally as a gel or a cream) against $\mathrm{HIV}^{21,22}$, we tested PS fibers which were coated with respectively poly(allylamine hydrochloride) (PAH, a cationic polyelectrolyte) or dextran sulfate sodium (DSS, a polyanionic low molecular weight derivative of dextran (Figure 1B)). We then compared the ability of neutral (PS), cationic (PAH-PS) or anionic (DSS-PS) fibers to bind HIV under conditions mimicking the healthy vaginal environment. Finally we evaluated whether the bound HIV particles were no longer able to infect $\mathrm{CD} 4^{+}$target cells.

\section{Materials and Methods}

\section{Materials.}

Dimethylformamide (DMF), tetrahydrofuran (THF), poly(allylamine hydrochloride) (PAH) and dextran sulfate sodium (DSS) were purchased from Sigma-Aldrich (Steinheim, Germany). Polystyrene (PS; $\mathrm{M}_{\mathrm{W}} 100000 \mathrm{~g} / \mathrm{mol}$ ) was purchased from Alfa Aesar (Karlsruhe, Germany). Polypropylene (PP) electrospun fibers were donated as a kind gift from Prof. Martin Möller (DWI an der RWTH, Aachen, Germany).

\section{Virus.}

HIV-1 subtype B, CCR5 co-receptor using reference strain Ba-L (NIH AIDS Research \& Reference Reagent program, Rockville, US) M.D.), was used. A stock of cell-free virus was 
prepared by culturing HIV-1 Ba-L in PHA/IL-2 stimulated mononuclear cells and tittered at 10,000 TCID 50 per mL. For some experiments, the virus was inactivated by incubation with $200 \mu \mathrm{M}$ Aldrithiol-2 (AT2 in DMSO) for $1 \mathrm{~h}$ at $37^{\circ} \mathrm{C}$ ). Subsequently, virus was filtered over a $100 \mathrm{kDa}$ cut-off membrane (Millipore) to remove AT2 and was aliquotted and stored at $-80{ }^{\circ} \mathrm{C}$. This treatment modifies the essential $\mathrm{Zn}$-fingers in the nucleocapsid protein, but leaves the viral envelope intact, allowing normal binding. ${ }^{23}$

\section{Preparation and functionalization of fiber webs by electrospinning.}

PS fibers were obtained through the electrospinning of a PS solution (Figure 1A) as described previously. ${ }^{19}$ Typically, $0.3 \mathrm{mg}$ PS was dissolved in $1 \mathrm{~mL}$ of a solvent mixture (DMF/THF; $1 / 3$ ) and subsequently electrospun into fibers. A high voltage power supply (up to $40 \mathrm{kv}$ ), a syringe, a flat-tip needle and a grounded collector were used in the electrospinning device. Typically, electrospinning of a $1 \mathrm{~mL}$ PS solution was conducted at a $2.0 \mathrm{~mL} \mathrm{~h}^{-1}$ feeding rate using a syringe pump (Prosense B.V. NE 300 USA). The applied voltage was kept at $8.4 \mathrm{kV}$. PS fiber webs were deposited on the grounded collector.

To make functionalized fibers, electrospun PS fibers (approximately $10 \mathrm{mg}$ ) were immersed in concentrated sulfuric acid $(9.8 \mathrm{M}, 10 \mathrm{~mL})$ and stirred for 2 min. The fibers were then removed from the acid solution and washed in distilled water until the $\mathrm{pH}$ of the solution was approximately 7. To make positively charged fibers, sulfonated PS fibers (approximately 10 $\mathrm{mg}$ ) were immersed in $5 \mathrm{~mL}$ of a PAH solution $(2 \mathrm{mg} \mathrm{PAH} / \mathrm{mL}$ in $0.5 \mathrm{M} \mathrm{NaCl})$ for $15 \mathrm{~min}$ and then extensively washed in water. Negatively charged fibers were made by coating PAH fibers with DSS $(0.2 \mathrm{mg} / \mathrm{mL}$ in distilled water). At the end of the procedure all types of fibers were thoroughly washed in distilled water. Before being used for experiments, the fibers were air-dried on a glass slide.

\section{SEM.}

$0.1 \mathrm{mg}$ of a PS fiber web was deposited onto a silicon wafer and dried under a nitrogen stream, followed by sputtering with gold. SEM images were recorded with a Quanta 200 FEG scanning electron microscope operated at an acceleration voltage of $15 \mathrm{kV}$. 


\section{Contact angle measurements.}

Contact angles were measured with a Kruss Drop Shape Analysis System G10/DSA10 (Krüss GMBH, Hamburg, Germany) from video images, using the tangent method. The focus of the camera lens was set at 2 . The image was calibrated with a Hamilton calibration needle with a known diameter. A water droplet of $10 \mu \mathrm{L}$ was dosed (with a speed of $200 \mu \mathrm{L} / \mathrm{min}$ ) on the surface of the fibers.

\section{Preparation of Simulated Vaginal Fluid (SVF).}

The preparation of SVF was described earlier. ${ }^{24}$ Briefly, NaCl, $(3.51 \mathrm{~g}) \mathrm{KOH},(1.4 \mathrm{~g})$ $\mathrm{Ca}(\mathrm{OH})_{2},(0.222 \mathrm{~g})$ bovine serum albumin, $(0.018 \mathrm{~g})$ lactic acid, $(2 \mathrm{~g})$ acetic acid, $(1 \mathrm{~g})$ glycerol, $(0.16 \mathrm{~g})$ urea, $(0.4 \mathrm{~g})$ and glucose $(5 \mathrm{~g})$ were dissolved in $1 \mathrm{~L}$ of water. The $\mathrm{pH}$ of the solution was adjusted to 4.2 with hydrochloric acid.

\section{Cell culture.}

Immortalized human vaginal epithelial cells were VK2/E6E7 were kindly provided by Dr. R. Fichorova (Harvard Medical School, Boston, MA) and cultured in a humid atmosphere at $37^{\circ} \mathrm{C}$ and $5 \% \mathrm{CO}_{2}$. Cells were kept in keratinocyte serum-free medium (Gibco, Invitrogen, Belgium) supplemented with human recombinant EGF $\left(0.1 \mathrm{ng} \mathrm{mL}^{-1}\right)$, bovine pituitary extract (0.05 $\left.\mathrm{mg} \mathrm{mL}^{-1}\right)$ and calcium chloride $(0.4 \mathrm{mM})$ (Gibco, Invitrogen, Belgium). Cells were passaged when reaching $70-80 \%$ confluency and split $1 / 5$. TZMbl cells were obtained from NIH AIDS Research and Reference Reagent Program, Division of AIDS, NIAID, NIH (Bethesda, US) and were cultured in DMEM (Lonza) supplemented with 10\% FCS (Lonza), $50 \mathrm{mg} / \mathrm{mL}$ gentamicin (Lonza) and $200 \mathrm{mM} \mathrm{L}$-glutamine (Lonza). Cells were refreshed twice a week.

\section{Toxicity tests.}

To test the toxicity of PS, PAH and DSS (and fibers made from these polymers), vaginal epithelial cells (VK2 cells) or TZMbl cellss were seeded in 24-well plates (50.000 cells/well) and allowed to settle overnight. The cells were then exposed to solutions of respectively PS, $\mathrm{PAH}$, and DSS and electrospun fibers for $5 \mathrm{~h}$ at $37^{\circ} \mathrm{C}$. Cell viability was evaluated $24 \mathrm{~h}$ later 
by an MTT assay according to the manufacturer's instructions (Roche, Vilvoorde, Belgium) and compared to the viability of untreated control cells.

\section{Adsorption of inactive HIV to fibers.}

Electrospun PS fibers, either naked, PAH-coated or DSS-coated (at varying concentrations (0-5 mg)) were incubated for $2 \mathrm{~h}$ in $200 \mu \mathrm{L}$ of a dispersion of inactive HIV (solvent respectively SVF or PBS). The concentration of inactive HIV in the dispersion (expressed as HIV-p24 antigen) equaled $220 \mathrm{pg}$ p24/mL, as measured by a commercial ELISA kit (InnoGenetics, Zwijnaarde, Belgium).All samples were kept in the dark at room temperature during incubation. After incubation, the p24 concentration in the supernatant (coming from non adsorbed HIV) was measured.

\section{Labeling of HIV.}

Inactivated HIV was covalently linked with NHS-alexa488 (Molecular Probes, Invitrogen, Belgium) according to the manufacturer's recommendations. For the labeling of $50 \mu \mathrm{L}$ inactivate HIV stock dispersion $(660 \mathrm{ng}$ p24/mL), $3 \mu \mathrm{L}$ of NHS-alexa488 was used. The excess of dye was removed by overnight dialysis against $5 \mathrm{~L}$ of distilled water using a Spectra/Por microdispo dialyser (Spectrumlabs, CA, USA) with a molecular weight cut off of $25 \mathrm{kD}$.

\section{Confocal microscopy.}

The binding of inactivate HIV to the fibers was further studied by confocal microscopy. To this end, $0.1 \mathrm{mg}$ of PAH-coated fibers was incubated in $1 \mathrm{~mL}$ of inactivate (fluorescently tagged) HIV (220 pg p24/mL) for $30 \mathrm{~min}$ at $37^{\circ} \mathrm{C}$. Afterwards the fibers were washed 3 times with PBS to remove unbound HIV. Subsequently, specific regions of the fibers were photobleached and imaged using a Biorad MRC 1024 confocal system. An inverted microscope (Eclipse TE300D, Nikon) equipped with a 20x and 40x objective lens was used.

\section{AFM measurements.}


For AFM measurements on free inactivate HIV, $5 \mu \mathrm{L}$ of a diluted HIV dispersion (6.6 ng p24/mL) was applied onto a silicon wafer. For AFM measurements on fibers, $1 \mathrm{mg}$ of PAH coated PS fibers was dispersed in $1 \mathrm{~mL}$ inactivate HIV dispersion $(6.6 \mathrm{ng} \mathrm{p} 24 / \mathrm{mL})$ for 1 hour at room temperature after which the fibers were washed three times with PBS to remove free HIV. Subsequently the fibers were deposited onto a silicon wafer and studied by AFM (Veeco, Mannheim, Germany); the AFM instrument was equipped with an optical microscope, a video camera, a monitor and AFM tip. Standard V-shaped 115- $\mu$ m-long silicon nitride cantilevers (with a spring constant of $0.32 \mathrm{~N} / \mathrm{m}$ ) and pyramidal tips (with an estimated tip diameter of 10 nm; DNP-S tips, Veeco) were used. The images were recorded in the tapping mode with 512 lines per screen and at a scan rate of $1.5 \mathrm{~Hz}$.

\section{HIV infection assay.}

Various amounts (as indicated in the main text) of electrospun PS fibers (either naked, PAH-coated or DSS-coated) were dispersed in $200 \mu \mathrm{L}$ of active HIV (220 pg p24/mL) in SVF or PBS and incubated in 96-well plates for $2 \mathrm{~h}$. All samples were kept in the dark at $37^{\circ} \mathrm{C}$. The viral efficacy was then determined making use of $\mathrm{CD}^{+}$TZMbl cells expressing luciferase under the control of an LTR promoter, as described in detail elsewhere. ${ }^{25}$

\section{Results}

\subsection{PS and PP electrospun fibers.}

As explained in the introduction, the avid binding of HIV particles to PS and PP Eppendorf tubes drove us to test HIV binding to electrospun PS and PP fibers. Representative scanning electron microscopy (SEM) images of PS and PP fibers are shown in Figure 2A, B. Average diameters were calculated to be 4.1 and $1.3 \mu \mathrm{m}$ for PS and PP fibers, respectively

(Figure 2C, D). Interestingly, whereas PP fibers showed a very smooth surface (Figure 2B), PS fibers were found to have a high porosity (Figure 2A), which may be explained by the differences in how the fibers were produced, as PS fibers were electrospun using a solution of PS in THF/DMF, while PP fibers were obtained through electrospinning of a PP melt. As one can expect that the high porosity increases the specific surface area, which may promote HIV binding, in further experiments, only the electrospun PS fibers were used. To evaluate the 
effect of surface charge on HIV binding, PS fibers were coated with polycationic PAH or polyanionic DSS, as described in the Materials and Methods section. The amount of PAH or DSS adsorbed onto the fibers could be calculated by measuring the concentration of either compound in solution before and after exposure to the fibers. For instance, for DSS coating, $10 \mathrm{mg}$ of PAH-coated fibers were suspended in a DSS-solution $(200 \mu \mathrm{g} / \mathrm{mL})$ for $15 \mathrm{~min}$ which resulted in a total of $2.5 \mu \mathrm{g}$ of DSS being adsorbed per mg of PAH-coated fibers.

To further evaluate the binding of PAH or DSS to the PS fibers, water contact angles were measured (Figure 3E, F, G), showing a clear decrease of the water contact angle for PAH $\left(11^{\circ}\right)$ and DSS $\left(25^{\circ}\right)$ coated fibers, when compared to naked PS $\left(130^{\circ}\right)$ fibers. These data confirm the adsorption of PAH and DSS to the PS fibers. The increased hydrophilicity of thefibers may facilitate the binding of HIV viral particles.

\subsection{Adsorption of inactive HIV to electrospun PS fibers.}

Next, the adsorption of inactive HIV to naked, PAH- and DSS-coated fibers was measured by means of a viral p24 specific ELISA assay. To this end, various amounts of the fibers were exposed (for $2 \mathrm{~h}$ ) to SVF containing $220 \mathrm{pg}$ p24/mL of inactive HIV. A concentration-dependent adsorption of HIV viral particles to all types of fibers was observed (Figure 3A). PAH- and DSS-coated fibers had similar binding efficiencies, which were slightly better than that for the more hydrophobic "naked" PS fibers. As both DSS- and PAH-coated fibers resulted in similar HIV-adsorption efficiencies, this may suggest that HIV viral particles contain both positive and negative charges. As viruses can have a lipid "envelope" derived from the host cell membrane, an overall negative charge of the particles has been reported, though at lower $\mathrm{pH}$ the overall negative surface charge diminishes. ${ }^{26}$ Furthermore, although phospholipid head groups such as phosphocholine (which is often present in natural membranes) have an overall neutral charge, the specific orientation of the negative and positive charges present may lead to local charge heterogeneities. Also, the viral envelope consists of several glycoproteins such as gp120, which includes several regions such as the V3 loop, which is known to contain high numbers of positively charged amino acids,

resulting in local positive charges. ${ }^{27}$ Taken together, the presence of both positive and negative charges on the HIV surface likely results in the better adsorption of HIV to the 
coated PS fibers compared to the neutral (non-coated) ones.

As during sexual intercourse slightly alkaline seminal fluid, which has a high buffering capacity, has been described to transiently elevate the $\mathrm{pH}$ of the surrounding vaginal fluid, ${ }^{28}$ we further tested the binding of HIV to fibers at a higher $\mathrm{pH}$ value. Inactive HIV particles were therefore suspended in phosphate buffered saline (PBS) with a $\mathrm{pH}$ of 7.0. The data in Figure 3B again clearly show an avid binding of the HIV viral particles to the various fibers. The binding efficiencies of bare, PAH- and DSS-coated PS fibers seemed rather similar. Interestingly, however, is the observation that in both conditions (SVF or PBS) the adsorption efficiency of the viral particles could be boosted up to nearly $100 \%$ upon using higher concentrations of the fibers.

\subsection{Direct analysis of inactive HIV binding to PAH-coated PS fibers.}

As PAH-coated fibers resulted in avid adsorption of inactive HIV viral particles in SVF, we tried to further characterize the binding through atomic force microscopy (AFM). The AFM data (Figure 4A) showed a flat surface with some indentations indicative of the high porosity of the fibers. AFM on (free) inactive HIV viral particles showed an average diameter of approximately $25 \mathrm{~nm}$ (Figure 4B). This value is significantly lower than the size $(120 \mathrm{~nm}$ ) reported by McPherson et al. ${ }^{29}$ and might be due to the chemical inactivation of the HIV viral particles which may result in a collapse of the viral core. Nonetheless, these particles were found to avidly bind to the PAH-coated fibers (Figure 4C, D).

To test whether the interaction between the HIV particles and the fibers was only transient or long-lasting, inactivate HIV was labeled with NHS-Alexa Fluor488 as described in the Materials and Methods section. These fluorescent viral particles were then incubated with PAH-coated fibers at room temperature for $1 \mathrm{~h}$, after which the fibers were washed 3 times with PBS and placed in SVF. Using an argon-ion laser beam at $488 \mathrm{~nm}$, fluorescent viral particles were bleached by spatial selective photobleaching at precise locations on the fibers (Figure 4E). These bleached fibers were subsequently kept in SVF for up to 4 days, after which the fibers were again visualized by confocal laser scanning microscopy (CLSM; Figure 4F). The data clearly show that the photobleached regions remain clear after 4 days, suggesting that the viral particles did not desorb and were firmly bound to the fibers, as any 
movement of viral particles along the fiber surface would have resulted in a recovery of the fluorescence in the bleached region.

\subsection{Cytotoxicity of electrospun fibers to vaginal epithelial cells.}

For an efficient inhibition of HIV transmission, electrospun fibers should be applied intravaginally. Of great important for maintaining the healthy vaginal environment is the vaginal epithelial lining. Any loss of cells or disruptions of the epithelial barrier function would greatly facilitate HIV transfer across the epithelial lining into deeper tissues and finally the blood stream, from where HIV could be spread throughout the body. To evaluate whether the fibers were toxic for vaginal epithelial cells, an MTT assay was performed on vaginal epithelial cells exposed to bare, PAH- or DSS-coated PS fibers (Figure 5A). The data show that DSS-coated and bare PS fibers did not have significant effects on mitochondrial metabolism. For bare PS fibers, a slight decrease in cell viability was noted at higher fiber concentrations. PAH-coated fibers resulted in a concentration-dependent decrease in cell viability. The toxicity of PAH-coated fibers is likely explained by the polycationic nature of PAH which may significantly interact with the anionic cell membrane thereby rupturing the cell membrane. Similar effects have been observed for other polycationic molecules such as poly-L-lysine. ${ }^{30}$

\subsection{Reduction of HIV infectivity by adsorption to electrospun PS fibers.}

The previous sections showed avid binding of inactive HIV viral particles to the electrospun fibers at different $\mathrm{pH}$. An important question which remains is whether the binding of active viral particles also lowers viral infection. Therefore, active HIV viral particles were exposed for $2 \mathrm{~h}$ to electrospun PS fibers dispersed in cell medium. Subsequently, samples of the cell medium were taken and infectivity (due to remaining HIV) was evaluated using $\mathrm{CD} 4^{+} \mathrm{TZMbl}$ cells, as described in the Materials and Methods section

(Figure 6B). The data clearly show a fiber concentration dependent reduction of the HIV infectivity. In line with the binding efficiency of inactive HIV viral particles to the various fibers, the effect was most pronounced for PAH-coated fibers, followed closely by DSS-coated ones. Bare PS fibers also reduced HIV infectivity, but to a lower extent. 
Interestingly, at the highest concentration of the fibers, infection of the cells by HIV could be almost completely abolished.

\section{Discussion}

Since HIV is characterized by a very high genetic variability, no vaccine is currently available. For that reason, novel strategies preventing HIV infection are highly required. One strategy which is currently widely investigated, is the use of microbicides ${ }^{31,32}$ One example of an intravaginal microbicidal gel is VivaGel ${ }^{\circledR}, 33$ which is currently being tested for its potential to prevent sexually transmitted infections (STIs), including HIV infection. However, all gel-based microbicides suffer from several practical inconveniences such as the need to clean and reuse the gel applicators and washing away of the gel with urination. ${ }^{34}$ Recently, multiple large-scale clinical trials failed to show any effect of currently available microbicides, possibly due to the inability of current drug formulations to form a stable and durable barrier along the whole epithelial lining. ${ }^{33}$ In order to try and overcome these issues, researchers are exploring the use of nanotechnology, such as e.g. polystyrene nanoparticles coated with lectins, such as Concanavalin A which is reported to have a high affinity for the viral protein gp120. ${ }^{35}$ Another study reported on the use of mercaptobenzoic acid modified $2 \mathrm{~nm}$ diameter Au nanoparticles which were conjugated to SDC-1721, which is a known antagonist of CCR5 and may thus be of use to prevent HIV infection. ${ }^{36}$ Such nanoparticles, however, still suffer from the same intrinsic problems as the gel-based microbicide formulations, i.e. they do not form a durable barrier along the entire epithelial lining and would need to be reapplied frequently.

Another possible strategy relies on the intravaginal application of effective adsorptive films. An adsorptive film is a sort of a woman's condom that could prevent transmission of HIV by virus adsorption and inactivation. The present work takes a first step in this direction, where electrospun fibers were found to efficiently bind and inactivate HIV viral particles. Moreover, from such fibers a 'web-based' film can be produced which may be applied as a condom. The high specific surface area and porosity of the electrospun PS fibers allow for a high binding potential which may offer great potential in the efficient capture of HIV. The flexibility of the electrospinning process furthermore may allow to easily incorporate 
anti-viral drugs in the fibers to further boost the antiviral efficacy. ${ }^{37}$ Another advantage of PS fibers is their relative low cost compared to the expensive antiviral drugs and the ability to upscale production.

\section{Conclusions}

Based on our initial observations that inactivate HIV viral particles avidly bound to PS and PP Eppendorf tubes at low $\mathrm{pH}$, the binding of the HIV to electrospun PS fibers was investigated. The electrospun PS fibers were characterized and showed a highly porous structure. Coating of the PS fibers with PAH or DSS resulted in charged fibers with increased hydrophilicity. Interestingly, the coated fibers were found to have even higher HIV binding efficiencies than naked PS fibers. Using AFM the binding of the viral particles to PAH fibers could be directly demonstrated while fluorescence recovery after photobleaching experiments revealed a strong binding. Naked and DSS-coated fibers were not toxic to vaginal epithelial cells while PAH-coated fibers affected their viability. Finally we showed that the coated PS fibers efficiently captured HIV thereby avoiding the infection of $\mathrm{CD} 4^{+} \mathrm{TZMb} 1$ cells.

\section{Acknowledgments}

We thank Prof. Martin Möller (DWI an der RWTH, Aachen, Germany) for his kind donation of electrospun PP fibers. SJS is a post-doctoral fellow from the FWO-Vlaanderen. Financial support from Ghent University is greatly acknowledged.

\section{References}

1. Greiner, A.; Wendorff, J. H., Electrospinning: A fascinating method for the preparation of ultrathin fibres. Angew Chem Int Edit 2007, 46 (30), 5670-5703.

2. Sill, T. J.; von Recum, H. A., Electrospinning: applications in drug delivery and tissue engineering. Biomaterials 2008, 29 (13), 1989-2006.

3. Kumbar, S. G.; James, R.; Nukavarapu, S. P.; Laurencin, C. T., Electrospun nanofiber scaffolds: engineering soft tissues. Biomed Mater 2008, 3 (3), 034002.

4. Huang, C.; Soenen, S. J.; Rejman, J.; Lucas, B.; Braeckmans, K.; Demeester, J.; De Smedt, S. C., Stimuli-responsive electrospun fibers and their applications. Chem Soc Rev 2011.

5. Li, D.; Wang, Y. L.; Xia, Y. N., Electrospinning nanofibers as uniaxially aligned arrays and layer-by-layer stacked films. Adv Mater 2004, 16 (4), 361-366.

6. Li, D.; Xia, Y. N., Electrospinning of nanofibers: Reinventing the wheel? Adv Mater 2004, 16 (14), 1151-1170. 
7. Ji, W.; Sun, Y.; Yang, F.; van den Beucken, J. J.; Fan, M.; Chen, Z.; Jansen, J. A., Bioactive electrospun scaffolds delivering growth factors and genes for tissue engineering applications. Pharm Res 2011, 28 (6), 1259-72.

8. Gillette, B. M.; Rossen, N. S.; Das, N.; Leong, D.; Wang, M.; Dugar, A.; Sia, S. K., Engineering extracellular matrix structure in 3D multiphase tissues. Biomaterials 2011, 32 (32), 8067-76.

9. Kurpinski, K.; Patel, S., Dura mater regeneration with a novel synthetic, bilayered nanofibrous dural substitute: an experimental study. Nanomedicine (Lond) 2011, 6 (2), 325-37.

10. Chew, S. Y.; Mi, R.; Hoke, A.; Leong, K. W., Aligned Protein-Polymer Composite Fibers Enhance Nerve Regeneration: A Potential Tissue-Engineering Platform. Adv Funct Mater 2007, 17 (8), 1288-1296.

11. Saraf, A.; Baggett, L. S.; Raphael, R. M.; Kasper, F. K.; Mikos, A. G., Regulated non-viral gene delivery from coaxial electrospun fiber mesh scaffolds. Journal of controlled release : official journal of the Controlled Release Society 2010, 143 (1), 95-103.

12. Szentivanyi, A.; Chakradeo, T.; Zernetsch, H.; Glasmacher, B., Electrospun cellular microenvironments: Understanding controlled release and scaffold structure. Adv Drug Deliver Rev 2011, 63 (4-5), 209-20.

13. Sefcik, L. S.; Neal, R. A.; Kaszuba, S. N.; Parker, A. M.; Katz, A. J.; Ogle, R. C.; Botchwey, E. A., Collagen nanofibres are a biomimetic substrate for the serum-free osteogenic differentiation of human adipose stem cells. J Tissue Eng Regen Med 2008, 2 (4), 210-20.

14. Xie, J.; Willerth, S. M.; Li, X.; Macewan, M. R.; Rader, A.; Sakiyama-Elbert, S. E.; Xia, Y., The differentiation of embryonic stem cells seeded on electrospun nanofibers into neural lineages. Biomaterials 2009, 30 (3), 354-62.

15. Xie, J.; Macewan, M. R.; Willerth, S. M.; Li, X.; Moran, D. W.; Sakiyama-Elbert, S. E.; Xia, Y., Conductive Core-Sheath Nanofibers and Their Potential Application in Neural Tissue Engineering. Adv Funct Mater 2009, 19 (14), 2312-2318.

16. Choi, S. H.; Hwang, I. S.; Lee, J. H.; Oh, S. G.; Kim, I. D., Microstructural control and selective $\mathrm{C}(2) \mathrm{H}(5) \mathrm{OH}$ sensing properties of $\mathrm{Zn}(2) \mathrm{SnO}(4)$ nanofibers prepared by electrospinning. Chem Commun 2011, 47 (33), 9315-7.

17. Ding, B.; Wang, X. F.; Yu, J. Y.; Wang, M. R.; Pan, F. K., A highly sensitive humidity sensor based on a nanofibrous membrane coated quartz crystal microbalance. Nanotechnology 2010, 21 (5).

18. Liu, Y.; Rafailovich, M. H.; Malal, R.; Cohn, D.; Chidambaram, D., Engineering of bio-hybrid materials by electrospinning polymer-microbe fibers. Proc Natl Acad Sci U S A 2009, 106 (34), 14201-6.

19. Huang, C. B.; Lucas, B.; Vervaet, C.; Braeckmans, K.; Van Calenbergh, S.; Karalic, I.; Vandewoestyne, M.; Deforce, D.; Demeester, J.; De Smedt, S. C., Unbreakable Codes in Electrospun Fibers: Digitally Encoded Polymers to Stop Medicine Counterfeiting. Adv Mater 2010, 22 (24), 2657-+.

20. Varshosaz, J.; Jannesari, M.; Morshed, M.; Zamani, M., Composite poly(vinyl alcohol)/poly(vinyl acetate) electrospun nanofibrous mats as a novel wound dressing matrix for controlled release of drugs. Int J Nanomed 2011, 6. 
21. Ariën KK, Jespers V, Vanham G. HIV sexual transmission and microbicides. Rev Med Virol 2011, 21: 110-133.

22. McGowan I., Microbicides for HIV prevention: reality or hope? Curr Opin Infect Dis 2010, 23(1): 26-31.

23. Rossio JL, Esser MT, Suryanarayana K, Schneider DK, Bess JW Jr, Vasquez GM, Wiltrout TA, Chertova E, Grimes MK, Sattentau Q, Arthur LO, Henderson LE, Lifson JD., Inactivation of human immunodeficiency virus type 1 infectivity with preservation of conformational and functional integrity of virion surface proteins. J Virol. 1998, 72(10), $7992-8001$.

24. Owen, D. H.; Katz, D. F., A vaginal fluid simulant. Contraception 1999, 59 (2), 91-95.

25. Jay, J. I.; Lai, B. E.; Myszka, D. G.; Mahalingam, A.; Langheinrich, K.; Katz, D. F.; Kiser, P. F., Multivalent benzoboroxole functionalized polymers as gp120 glycan targeted microbicide entry inhibitors. Mol Pharm 2010, 7 (1), 116-29.

26. Lai, S. K.; Hida, K.; Shukair, S.; Wang, Y. Y.; Figueiredo, A.; Cone, R.; Hope, T. J.; Hanes, J., Human immunodeficiency virus type 1 is trapped by acidic but not by neutralized human cervicovaginal mucus. J Virol 2009, 83 (21), 11196-200.

27. Clapham, P. R.; McKnight, A., HIV-1 receptors and cell tropism. Brit Med Bull 2001, 58, 43-59.

28. TeviBenissan, C.; Belec, L.; Levy, M.; SchneiderFauveau, V.; Mohamed, A. S.; Hallouin, M. C.; Matta, M.; Gresenguet, G., In vivo semen-associated $\mathrm{pH}$ neutralization of cervicovaginal secretions. Clin Diagn Lab Immun 1997, 4 (3), 367-374.

29. McPherson, A.; Kuznetsov, Y. G.; Victoria, J. G.; Robinson, W. E., Atomic force microscopy investigation of human immunodeficiency virus (HIV) and HIV-Infected lymphocytes. J Virol 2003, 77 (22), 11896-11909.

30. Hategan, A.; Law, R.; Kahn, S.; Discher, D. E., Adhesively-tensed cell membranes: lysis kinetics and atomic force microscopy probing. Biophys $J$ 2003, 85 (4), 2746-59.

31. Pauwels, R.; DeClercq, E., Development of vaginal microbicides for the prevention of heterosexual transmission of HIV. J Acq Immun Def Synd 1996, 11 (3), 211-221.

32. Neurath, A. R.; Strick, N.; Li, Y. Y.; Debnath, A. K., Cellulose acetate phthalate, a common pharmaceutical excipient, inactivates HIV-1 and blocks the coreceptor binding site on the virus envelope glycoprotein gp120. Bmc Infect Dis 2001, 1, art. no.-17.

33. das Neves, J.; Amiji, M. M.; Bahia, M. F.; Sarmento, B., Nanotechnology-based systems for the treatment and prevention of HIV/AIDS. Adv Drug Deliver Rev 2010, 62 (4-5), 458-477.

34. Neurath, A. R.; Strick, N.; Li, Y. Y., Water dispersible microbicidal cellulose acetate phthalate film. Bmc Infect Dis 2003, 3, -.

35. Akashi, M.; Niikawa, T.; Serizawa, T.; Hayakawa, T.; Baba, M., Graft copolymers having hydrophobic backbone and hydrophilic branches part XIV - Capture of HIV-1 gp120 and virions by lectin-immobilized polystyrene nanospheres. Bioconjugate Chem 1998, 9 (1), 50-53.

36. Bowman, M. C.; Ballard, T. E.; Ackerson, C. J.; Feldheim, D. L.; Margolis, D. M.; Melander, C., Inhibition of HIV fusion with multivalent gold nanoparticles. J Am Chem Soc 2008, 130 (22), 6896-+.

37. Chunder, A.; Sarkar, S.; Yu, Y.; Zhai, L., Fabrication of ultrathin polyelectrolyte fibers 
and their controlled release properties. Colloids Surf B Biointerfaces 2007, 58 (2), 172-9. 


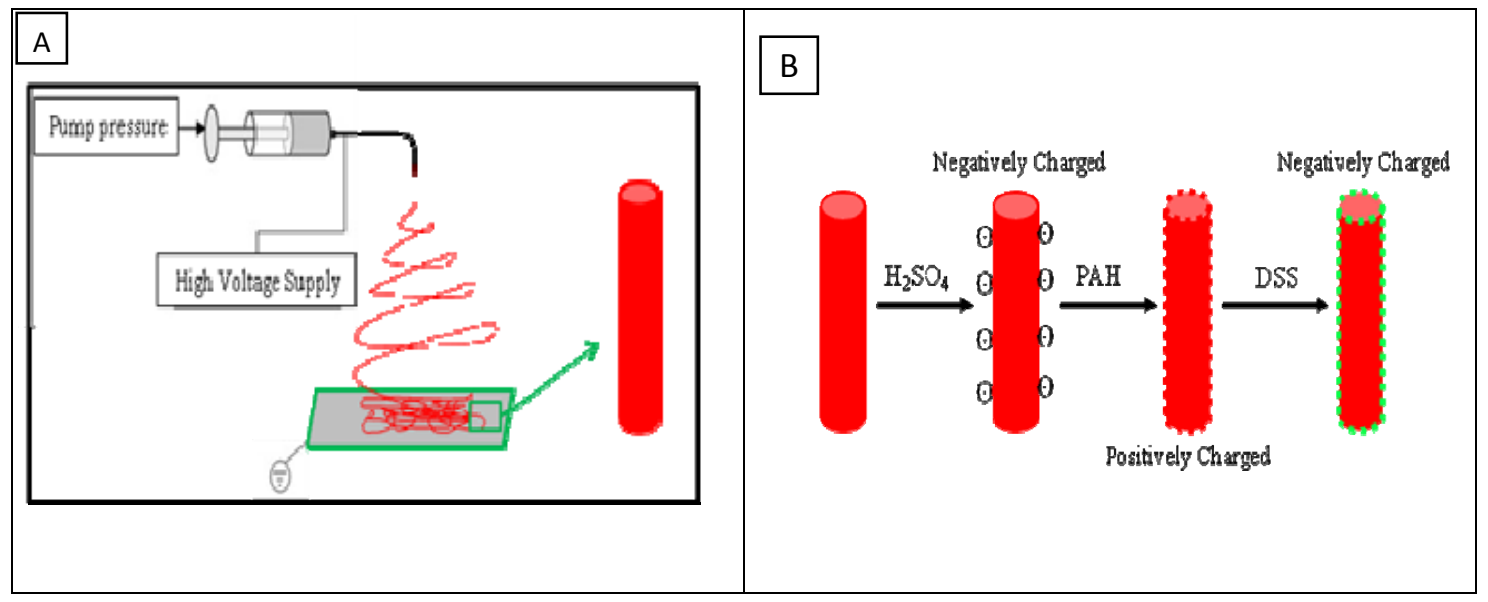

Figure 1. Schematic representation of the electrospinning setup and fiber functionalization. A) Electrospinning setup. B) Functionalization of electrospun fiber webs with $\mathrm{H}_{2} \mathrm{SO}_{4}$ to generate sulfonated fibers, after which the fibers can be covered with the polyelectrolyte PAH resulting in a positive surface (red). , These positive fibers can then be further functionalized through electrostatic interactions with PAH and DSS, resulting in a DSS (negatively charged) surface (green). 


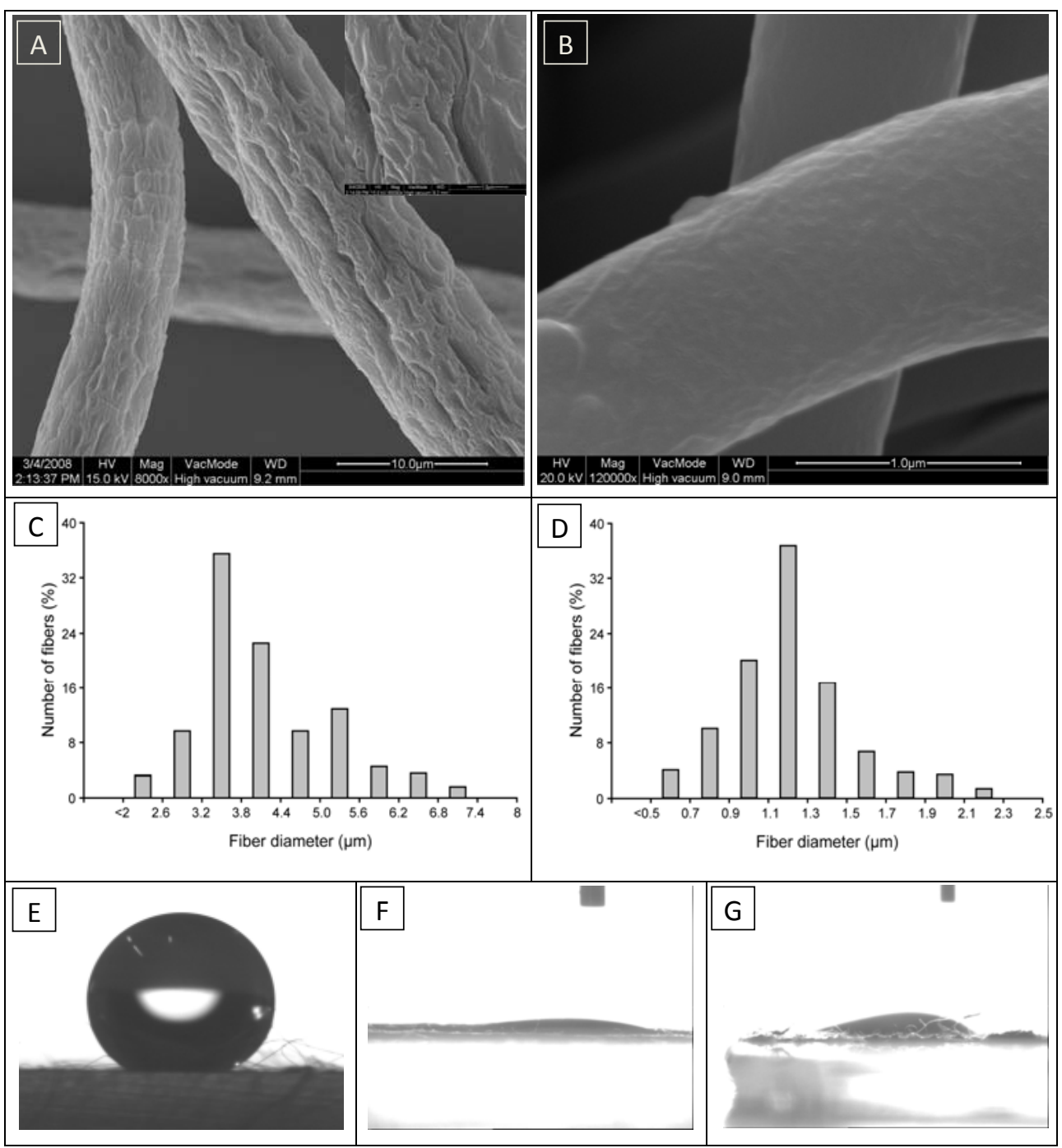

Figure 2. A) SEM image of PS electrospun fibers, the insert is a high magnification image. Scale bar: $10 \mu \mathrm{m}$. B) SEM image of PP electrospun fibers (as obtained from a melt). Scale bar: $1 \mu \mathrm{m}$. C, D) Histograms indicating the distribution of fiber diameters for PS fibers (C) and PP fibers (D). E-G) Representative images showing the typical structure of a water drop deposited at the surface of the electrospun fibers. Naked PS fibers (E), PAH-coated PS fibers (F) and DSS-coated PS fibers (G). 


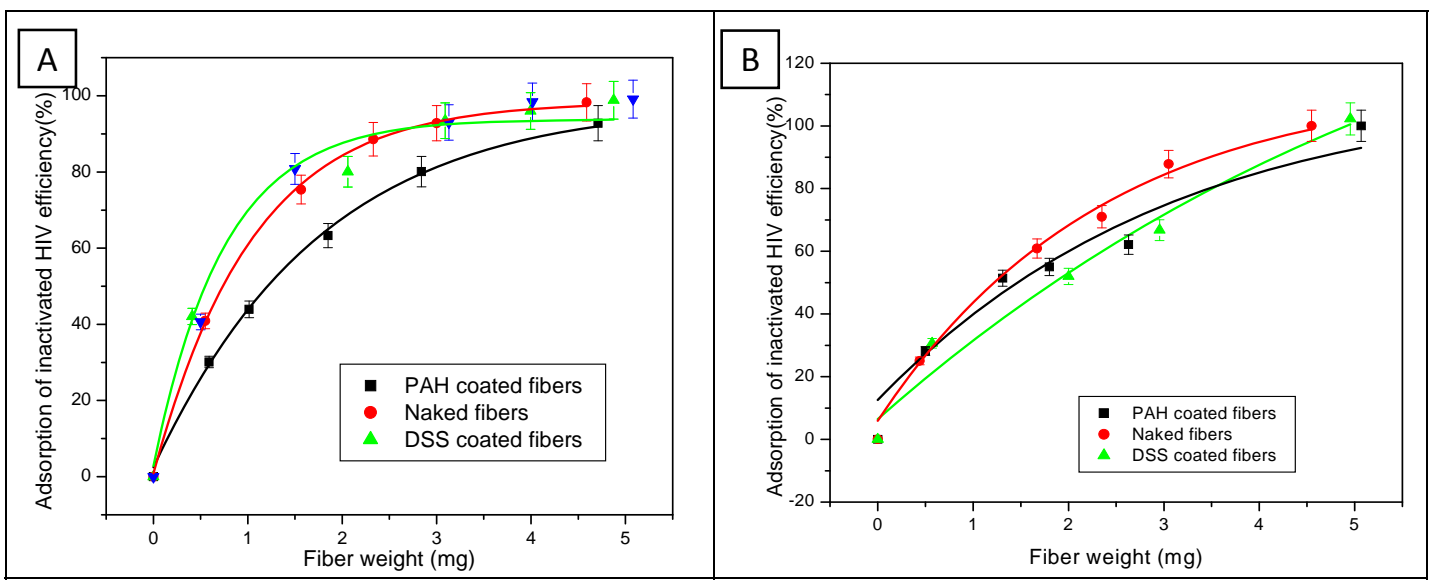

Figure 3. The binding of inactivate HIV-1 to three types of electrospun fibers (naked PS fibers (red), PAH-coated PS fibers (black) and DSS-coated PS fibers (green) at concentrations ranging from 0 to $5 \mathrm{mg} / \mathrm{ml}$ in respectively simulated vaginal fluid (A) and PBS (B). The results were obtained by measuring the p 24 concentration by an ELISA assay. Data are shown as mean $\pm \operatorname{SEM}(n=3)$. 


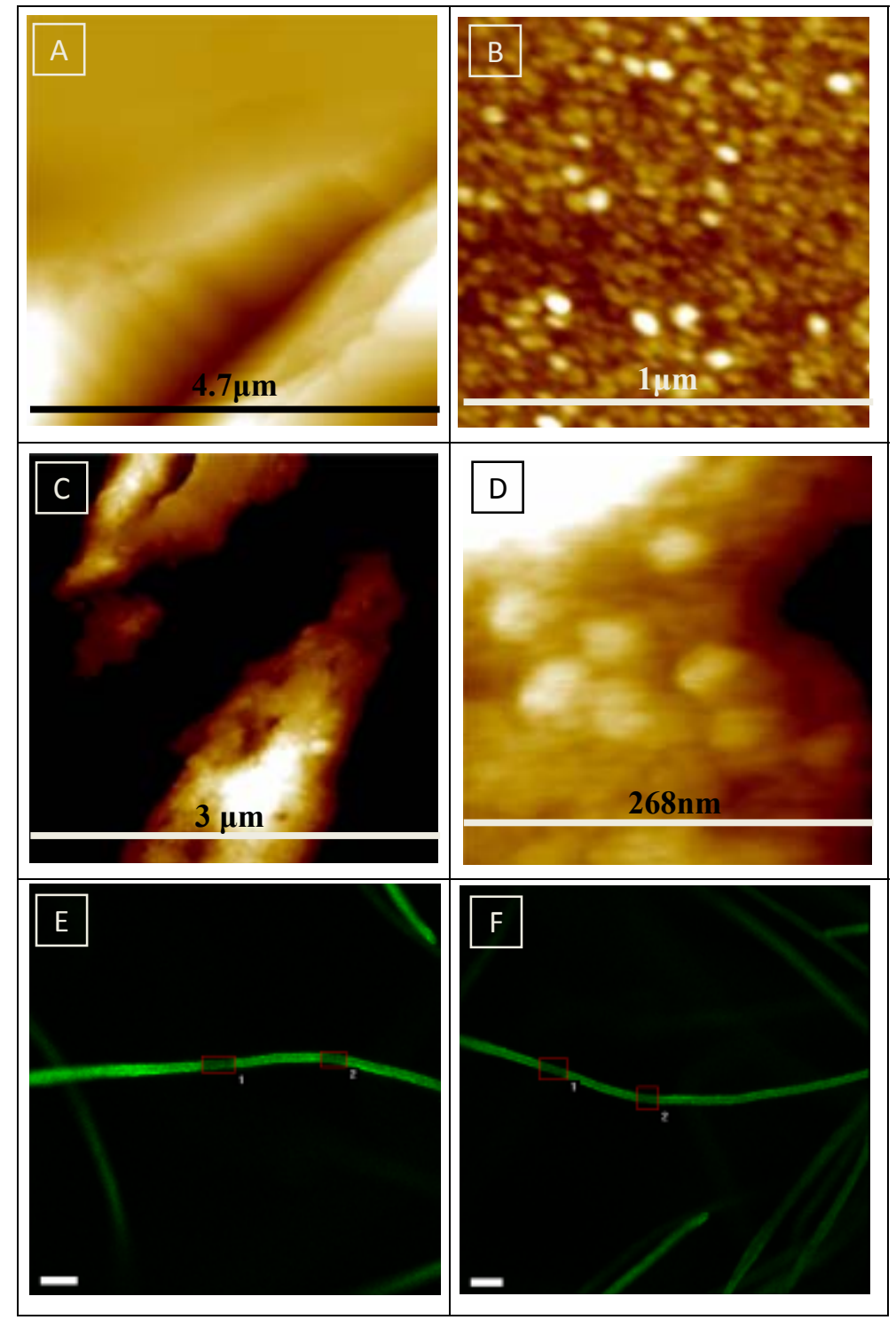

Figure 4. A-D) Atomic force microscopy images of A) PAH-coated PS fiber surface, B) inactivated HIV deposited on a silicon wafer, C) inactivated HIV adsorbed onto PAH-coated PS fibers, D) is a magnified view of image. E, F) Representative confocal fluorescence images of PAH-coated PS fibers incubated with fluorescently-tagged inactivate HIV (green). Regions 1 and 2 indicated by the red rectangles are areas which were bleached by spatial selective photobleaching. E) Representative image immediately after bleaching. F) Representative image after keeping the fibers for 4 days in SVF at room temperature. Scale bars: $20 \mu \mathrm{m}$. 


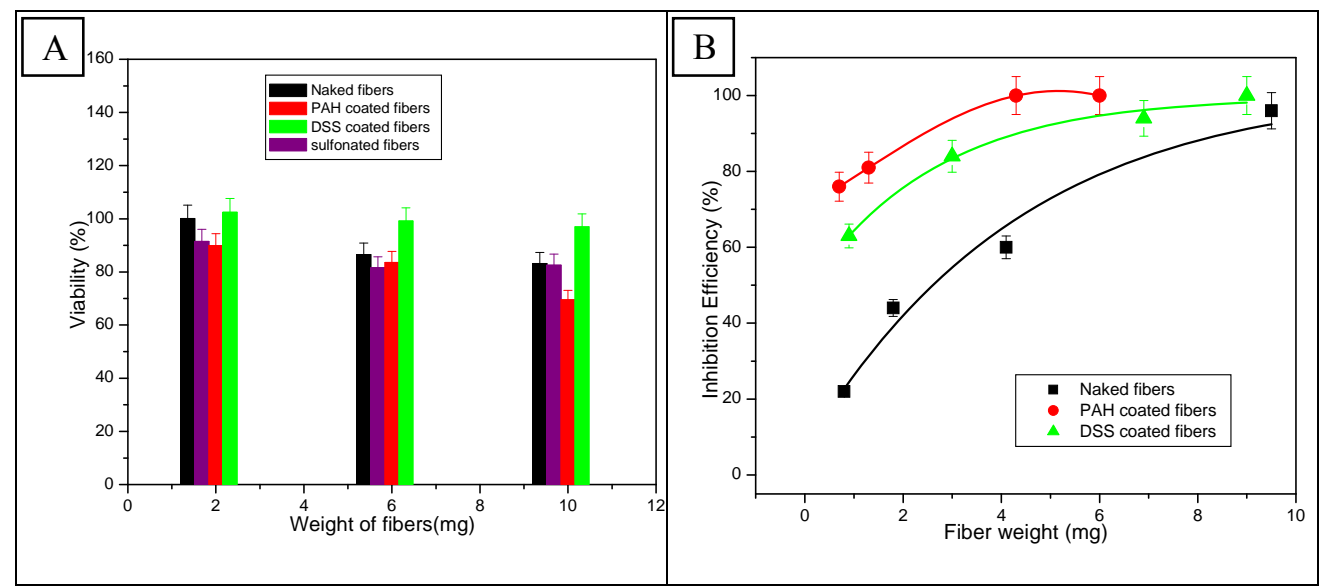

Figure 5. A) Viability of vaginal epithelial cells exposed to varying concentrations (2, 5 or 10 $\mathrm{mg} / \mathrm{ml}$ ) of naked PS fibers (black) and functionalized PS fibers (PAH coated (red), DSS coated (green) and sulfonated fibers (purple)). Data are shown as mean \pm SEM $(n=5)$. B) Inhibition of HIV-1 infection by electrospun PS fibers as determined by measuring the luminescence of $\mathrm{CD}^{+}$TZMbl target cells expressing luciferase. Active HIV was pre-incubated with three types of electrospun PS fibers (naked fibers (red), PAH coated fibers (black), DSS coated fibers (green)) for varying concentrations of the fibers after which samples of the supernatant were taken and incubated with vaginal endothelial cells. The infectivity of the viral particles is expressed as relative to that of viral particles which were treated similarly but not exposed to any fibers. Data are shown as mean $\pm \operatorname{SEM}(n=3)$ 


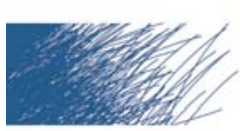

\section{$\widehat{\widehat{\text { IIIIII }}}$ UNIVERSITEIT GENT}

biblio.ugent.be

The UGent Institutional Repository is the electronic archiving and dissemination platform for all UGent research publications. Ghent University has implemented a mandate stipulating that all academic publications of UGent researchers should be deposited and archived in this repository. Except for items where current copyright restrictions apply, these papers are available in Open Access.

This item is the archived peer-reviewed author-version of : Electrospun polystyrene fibers for HIV entrapment

Authors: Chaobo Huang1,a, Stefaan J. Soenen2,a, Ellen van Gulck3, Joanna Rejman2, Guido Vanham3,4, Bart Lacus2, Bart Geers2, Kevin Braeckmans2, Victor Shahin5, Pieter Spanoghe6, Jo Demeester2, Stefaan C. De Smedt2*

In: Polymers Advanced Technologies 2014 (wileyonlinelibrary.com) DOI: 10.1002/pat.3310

Optional: link to the article

To refer to or to cite this work, please use the citation to the published version:

Authors (year). Title. journal Volume(Issue) page-page 